\title{
Reform based on Improving the Physical Fitness of College Students
}

\author{
Wang Ming \\ College of Sports Science, Zhuhai College of Jilin University, Jilin, China, 519041
}

Keywords: college students; healthy fitness; sports behavior; breakthrough

Abstract: In modern society, people's needs are not limited to the right to life and development, but to the pursuit of quality life. Correctly understanding, measuring and evaluating the state of quality of life will help humans to rationally plan and control life resources, promote physical and mental health and prolong life expectancy. Paying attention to the quality of life of college students, improving the overall level of their quality of life, and tapping the overall potential of the group will undoubtedly add a huge wealth to the country's construction.

\section{Introduction}

The main purpose of physical fitness is healthy body, quality life, and physical exercise is an effective means to improve physical fitness. This study intends to investigate the quality of life, physical fitness and exercise behavior of college students, analyze the status quo of quality of life and physical fitness and exercise behavior, and explore the relationship between quality of life and physical fitness and exercise behavior. Effectively improve the physical health of college students, and formulate relevant health promotion strategies to provide new breakthroughs to provide evidence.

\section{Objects and methods}

\subsection{The object adopts the stratified equivalence random sampling method}

Hebei University of Technology, Institute of Disaster Prevention Science and Technology, Lang fang Teachers College, Hebei Vocational and Technical College, and Hebei Normal University as the primary sampling units. School 2, 4 comprehensive universities; each university stratified 4 grades as sub-sampling units; each grade is randomly selected 40 classes as a three-level sampling unit according to the 5\% ratio standard; each administrative class adopts A random sampling plan of random numbers was used to extract 25 students as the minimum sampling unit. A total of 1,000 male and female college students were selected to form the sample. 956 valid questionnaires were collected, and the effective recovery rate was $95.6 \%$, including 524 boys and 432 girls ${ }^{[1]}$.

\subsection{Method}

The assessment tool for measuring the quality of life is the health survey summary SF-36 scale. 
There are 8 health dimensions in this survey, and the Cornbrash's $\alpha$ coefficient is $0.841 \sim 0$. Between 887. The scale uses the Likert accumulation method to divide the entries in the 8 dimensions into different equal-order variables. Since each item is graded differently, the original score is calculated according to the final value, and then the conversion score is calculated according to the standard formula:

$$
\text { Conversion score }=\frac{(\text { original score }- \text { lowest possible score })}{\text { possible score range }} \times 100
$$

Calculate the standard score of quality of life. Physical fitness includes cardiorespiratory fitness (4 items), muscle fitness ( 5 items), body composition (3 items), and flexibility (4 items). Exercises include exercise times, exercise time, and exercise intensity. The Cornbrash 's $\alpha$ coefficient of the three dimensions of exercise, time and intensity of the survey was $0.823,0.849,0.863^{[2]}$.

Table 1: Estimated values of physical fitness of college students

\begin{tabular}{ccc}
\hline \multirow{2}{*}{ Independent variable } & \multicolumn{2}{c}{ Body composition } \\
\cline { 2 - 3 } Exercise time & $\beta$ value & T value \\
Number of exercises & 0.230 & 3.109 \\
Exercise intensity & 0.159 & 1.921 \\
F value & -0.036 & -0.522 \\
R2value & & 7.094 \\
\hline
\end{tabular}

Table 2: Estimated values of cardiopulmonary fitness for college students

\begin{tabular}{|c|c|c|}
\hline \multirow{2}{*}{ Independent variable } & \multicolumn{2}{|c|}{ Cardiopulmonary fitness } \\
\hline & $\beta$ value & T value \\
\hline Exercise time & 0.212 & 2.791 \\
\hline Number of exercises & 0.149 & 1.842 \\
\hline Exercise intensity & -0.097 & -1.388 \\
\hline F value & \multicolumn{2}{|c|}{3.741} \\
\hline R2value & \multicolumn{2}{|c|}{0.061} \\
\hline
\end{tabular}

Table 3: Estimated values of muscle fitness in college students

\begin{tabular}{ccc}
\hline \multirow{2}{*}{ Independent variable } & \multicolumn{3}{c}{ Muscle fitness } \\
\cline { 2 - 4 } Exercise time & $\beta$ value & T value \\
Number of exercises & 0.124 & 2.162 \\
Exercise intensity & 0.173 & 2.282 \\
F value & 0.013 & 0.089 \\
R2value & & 3.454 \\
\end{tabular}

As shown in Table 1-3 above, using the Likert self-evaluation method, the lower factors or entries of the two variable sets are divided into five equal-order variables, which are recorded as 1 , $2,3,4$, and 5 points respectively. The scores of the fitness factors are calculated according to the standard formula. Considering that it is difficult for the subjects to measure the actual strength of the exercise with the professional concept, it is measured by the degree of sweating during exercise and self-evaluation of fatigue. 


\subsection{Statistical processing First analyze the various aspects of exercise behavior for healthy fitness}

Whether the regression is significant, and then uses the hierarchical regression analysis to investigate whether the influence of healthy fitness on the quality of life will be weakened after adding the exercise behavior, that is, the health fitness is the independent variable, and the quality of life is the dependent variable. Then, exercise behavior and healthy fitness are used as independent variables, and quality of life is the dependent variable for hierarchical regression analysis. The reverse-scoring entries are converted using Recode. The indicators were statistically processed using $(\mathrm{x} \pm \mathrm{s}), 95 \%$ CI normality test and homogeneity test of variance ${ }^{[3]}$.

\section{Results}

\subsection{Related analysis}

Results Table 1 shows that there are correlations between the dimensions of different quality of life and different physical fitness of college students, except for the emotional function dimension ( $\mathrm{P}<0.01$ or $\mathrm{P}<0.05$ ). In relation to exercise behavior, quality of life is positively correlated with exercise time and number of times, while some dimensions are negatively correlated with exercise intensity.

\section{2 regression analysis results}

\subsubsection{Regression analysis of exercise behavior and physical fitness}

It can be seen from the results in Table 2 that the two variables of exercise time and exercise time are the main predictors of physical fitness. The exercise times are mainly in the three dimensions of body composition, cardiopulmonary fitness and muscle fitness:

$\checkmark$ The beta value is at $0.149 \sim 0$. Between 173 ( $\mathrm{P}$ value is $<0.05$ );

The exercise time is shown in the four dimensions of healthy fitness:

$\checkmark$ The beta value is at $0.124 \sim 0$. Between $284(\mathrm{P}<0.05$ or $\mathrm{P}<0.01)$.

Although the regression of intensity variables to the dimensions of physical fitness did not reach statistical level, the variance explained in the four dimensions of healthy fitness was cumulatively 5 . $6 \%$ to 9 . Between $8 \%$, it is suggested that exercise behavior has a significant impact on physical fitness, mainly in the time and frequency of exercise ${ }^{[4]}$.

\subsubsection{Health fitness and quality of life and regression results after joining exercise behavior}

The data from Table 3 shows that the four dimensions of healthy fitness have different regression effects on the dimensions of life quality. The cumulative variance variance explained is the emotional function and mental health, followed by physiological function, physiological function and Vitality, the smaller is physical pain and overall health, the least impact on social function, statistically significant.

Table 4 above shows that healthy fitness has a strong predictive effect on the dimensions of quality of life. When considering physical fitness and exercise behavior at the same time, the regression coefficients between healthy fitness and quality of life dimensions were reduced, and even the impact on individual dimensions of quality of life was not statistically significant. In addition, after adding the exercise behavior variable, the variance explained is accumulated from the $\beta 1$ value of $13.6 \%$ to $28.3 \%$ increased to 18.2 . $8 \%$ to 84 . At $5 \%$, the F ratio reached a statistical level. The results of the data indicate that exercise behavior has a greater impact on the relationship 
between physical fitness and quality of life.

Table 4 Cranach $\alpha$ coefficient of the degree of conformity between college education

\begin{tabular}{|c|c|c|}
\hline $\begin{array}{c}\text { Cranach, s } \\
\text { Alpha }\end{array}$ & $\begin{array}{c}\text { Cranach, s } \\
\text { Alpha Based } \\
\text { On } \\
\text { Standardized } \\
\text { Items }\end{array}$ & $\mathrm{N}$ \\
\hline 0.708 & 0.716 & 25 \\
\hline
\end{tabular}

\section{Discussion}

\subsection{Lack of exercise behavior, healthy fitness and quality of life score is not high}

According to sports medicine research, physical exercise more than 3 times a week (including 3 times) and more than 30 minutes each time has the functions of improving physical health, enhancing physical fitness, preventing non-infectious diseases, relieving stress and improving mental health. Positive, this is also one of the criteria for dividing the sports population. However, the scores of the three factors of college students' sports behavior were below the median. In addition to the intensity of exercise, the differences between the other two factors were statistically significant. The four factors of health fitness and the total score of quality of life, in addition to flexibility, other factors scored statistically significant differences between male and female. The reason is that many aspects, this article thinks that subjectively, college students are not enough to understand the behavior of sports; objectively, subject to the factors such as venue equipment and extracurricular physical exercise, the root cause of serious imbalance between men and women is caused. Lack of motor behavior is an important factor leading to low fitness and quality of life scores.

\subsection{Exercise behavior has a greater impact on physical fitness}

The survey results show that exercise behavior has a strong predictive power for healthy fitness. Health fitness is an important concept that reflects the body's adaptability to the external environment. It is an important criterion for measuring the various qualities of the body. It is a physical adaptation and a dynamic ability. Scientific exercise is the use of reasonably designed exercise stimuli to break through the body's mechanisms of maintaining constantity, tempting the body to resist counteracting and adapting to the intense motor stimulation of the body and mind. Repeatedly, this situation will make the body have a strong ability to adapt to exercise stimuli. This is the improvement of healthy fitness. Tao Yong and other studies have shown that regular participation in exercise, the establishment of a good sports lifestyle, and the development of exercise habits can promote the development of various sports qualities of the body and play an important role in physical health.

\section{Conclusions}

It shows that on the basis of the greater contribution of physical fitness to quality of life, exercise behavior enhances the predictive power of healthy fitness to quality of life. The impact of physical fitness on the quality of life dimension is achieved entirely through motor behavior and can have a significant impact on physiological functions, social functions, emotional functions, vitality and mental health in quality of life. These effects are reduced by the addition of exercise behavior, 
indicating that the effects of healthy fitness on these five dimensions of life quality have both direct and indirect effects, and their indirect effects are achieved through exercise behavior.

\section{Acknowledgement}

Educational and Scientific Research Project of Guangdong Province: Research on the Reform of Physical Education Curriculum System in Colleges and Universities based on Health and Physical Fitness (No: jk012)

\section{References}

[1] Yi M, Long Z, Chen W, et al. The analysis on college students' physical fitness testing data - two cases study[C]// International Conference on Security. 2018.

[2] Zhou Z, Dong S, Yin J, et al. Improving Physical Fitness and Cognitive Functions in Middle School Students: Study Protocol for the Chinese Childhood Health, Activity and Motor Performance Study (Chinese CHAMPS).[J]. International Journal of Environmental Research \& Public Health, 2018, 15(5):976.

[3] Buresh R, Hornbuckle L, Garrett D, et al. Associations between measures of health-related physical fitness and cardiometabolic risk factors in college students. [J]. Journal of American College Health, 2018:00-00.

[4] Calestine J, Bopp M, Bopp C M, et al. College Student Work Habits are Related to Physical Activity and Fitness. [J]. International Journal of Exercise Science, 2017, 10(7):1009-1017. 